\title{
Heavy flavor operator matrix elements at $O\left(a_{s}^{3}\right)$
}

\author{
Isabella Bierenbaum; Johannes Blümlein, Sebastian Klein ${ }^{\dagger}$ \\ Deutsches Elektronen-Synchrotron, DESY \\ Platanenallee 6, D-15738 Zeuthen, Germany \\ E-mail: Isabella.Bierenbaum@ific.uv.es, \\ Johannes.Bluemlein@desy.de, Sebastian.Klein@desy.de
}

The heavy quark effects in deep-inelastic scattering in the asymptotic regime $Q^{2} \gg m^{2}$ can be described by heavy flavor operator matrix elements. Complete analytic expressions for these objects are currently known to NLO. We present first results for fixed moments at NNLO. This involves a recalculation of fixed moments of the corresponding NNLO anomalous dimensions, which we thereby confirm.

8th Conference Quark Confinement and the Hadron Spectrum

September 1-6, 2008

Mainz. Germany

* Present address: Instituto de Física Corpuscular, CSIC-Universitat de València, Apartado de Correros 22085, E46071 Valencia, Spain. Partially supported by the Ministerio de Ciencia e Innovación under Grant No. FPA2007-60323, by CPAN (Grant No. CSD2007- 00042), and by the European Commission MRTN FLAVIAnet under Contract No. MRTN-CT-2006-035482.

†Speaker.

${ }^{\ddagger}$ Supported in part by Studienstiftung des Deutschen Volkes. 


\section{Introduction}

The double-differential cross section for unpolarized deep inelastic scattering via single photon exchange can be expressed in terms of two structure functions $F_{2, L}\left(x, Q^{2}\right)$. Especially in the region of smaller values of $x$, these structure functions contain large $c \bar{c}$-contributions of up to 20-40\%, denoted by $F_{2, L}^{c \bar{c}}\left(x, Q^{2}\right)^{1}$. The perturbative heavy flavor Wilson coefficients $H_{2, L}\left(z, Q^{2}, m^{2}\right)$ corresponding to these structure functions are known at NLO semi-analytically in $z$-space, [1], with a fast numerical implementation in Mellin- $N$ space given in [2]. Due to the size of the heavy flavor corrections, it is necessary to extend the description of these contributions to $O\left(a_{s}^{3}\right), a_{s}:=\alpha_{s} /(4 \pi)$, and thus to the same level which has been reached for the massless Wilson coefficients, [3]. This will allow for a more precise determination of parton distribution functions and the QCD-scale $\Lambda$. A calculation of these quantities in the whole kinematic range at NNLO seems to be out of reach at present. However, in the limit of large virtualities $Q^{2}, Q^{2} \gtrsim 10 m_{c}^{2}$ in the case of $F_{2}^{c \bar{c}}\left(x, Q^{2}\right)$, one observes that $F_{2, L}^{c \bar{c}}\left(x, Q^{2}\right)$ are very well described by their asymptotic expressions, [ $₫$, neglecting power corrections in $m^{2} / Q^{2}$. In this kinematic range, one can calculate the heavy flavor Wilson coefficients analytically. This has been done for $F_{2}^{c \bar{c}}\left(x, Q^{2}\right)$ to 2-loop order in [ $₫$, 5] and for $F_{L}^{c \bar{c}}\left(x, Q^{2}\right)$ to 3-loop order in [6]. In the latter case, the asymptotic result becomes valid only at much higher values of $Q^{2}$. As first steps towards the 3-loop calculation at asymptotic scales for $F_{2}^{c \bar{c}}\left(x, Q^{2}\right)$ we calculated the $\mathrm{O}(\varepsilon)$ terms of the 2-loop heavy operator matrix elements (OMEs), ㄱ, \&], which contribute to the asymptotic 3-loop heavy flavor Wilson coefficients via renormalization. In the present paper, we report on new results concerning moments of the heavy OMEs $A_{Q(q) q}^{\mathrm{PS}}, A_{q q, Q}^{\mathrm{NS},+}$ and $A_{g q, Q}$ at 3-loops. In doing so, the fermionic terms of the even moments $N=2 \ldots 12$ of the corresponding NNLO anomalous dimensions given in [9] are confirmed in an independent calculation.

\section{Heavy Flavor Operator Matrix Elements}

In the following, we consider massive OMEs of the flavor-decomposed twist-2 operators between partonic states

$$
A_{k i}^{\mathrm{S}, \mathrm{NS}}\left(\frac{m^{2}}{\mu^{2}}, N\right)=\left\langle i\left|O_{k}^{\mathrm{S}, \mathrm{NS}}\right| i\right\rangle_{H}=\delta_{k, i}+\sum_{l=1}^{\infty} a_{s}^{l} A_{k i}^{\mathrm{S}, \mathrm{NS},(l)}\left(\frac{m^{2}}{\mu^{2}}, N\right) .
$$

Here, $\mathrm{S}$ and NS are the singlet and non-singlet contributions, respectively, $i$ denotes the outer onshell particle $(i=q, g)$ and $O_{k}$ stands for the quarkonic $(k=q)$ or gluonic $(k=g)$ operator emerging in the light-cone expansion. The subscript $H$ indicates that we require the presence of heavy quarks of one type with mass $m$, while $\mu$ is the renormalization scale. We work in Mellin-space, with the Mellin-variable denoted by $N$. The logarithmic terms in $\mathrm{m}^{2} / \mu^{2}$ are completely determined by renormalization and contain contributions of the anomalous dimensions of the twist-2 operators. Thus at NNLO the fermionic parts of the 3-loop anomalous dimensions calculated in Refs. [9] appear. All pole terms provide a check on our calculation. The single pole term allows for a first independent calculation of the terms $\propto T_{F}$ in the 3-loop anomalous dimensions.

As outlined in Ref. 汎, in the limit $Q^{2} \gg m^{2}$ one applies the massive renormalization group equation to obtain the factorization of the heavy flavor Wilson coefficients into a Mellin-convolution of

\footnotetext{
${ }^{1}$ We consider extrinsic heavy flavor production only.
} 
the light flavor Wilson coefficients with the corresponding heavy OMEs. The light flavor Wilson coefficients are known up to three loop order [3] and carry all the process dependence, whereas the heavy quark OMEs are universal, process-independent objects and contain all mass corrections to $H_{2, L}$ up to terms proportional to $\left(\mathrm{m}^{2} / Q^{2}\right)^{a}, a \geq 1$.

A related application of the heavy OMEs is given when using a variable flavor number scheme to describe parton densities including massive partons. The OMEs are then the transition functions when going from $n_{f}$ to $n_{f}+1$ flavors and thus one may define parton densities for massive quarks, see e.g. Ref. [10]. This is of particular interest for heavy quark induced processes at the LHC, such as $c \bar{s} \rightarrow W^{+}$at large enough scales $Q^{2}$. In this context, one may show as well that various sum rules follow from momentum conservation, [10], e.g:

$$
\left.A_{q q, Q}^{\mathrm{NS},+}\right|_{N=2}+\left.A_{Q(q) q}^{\mathrm{PS}}\right|_{N=2}+\left.A_{g q, Q}\right|_{N=2}=0 .
$$

Note, that in Eq. (2.2) two different PS contributions can be distinguished and we adopt the notation $A_{Q(q) q}^{\mathrm{PS}}:=A_{Q q}^{\mathrm{PS}}+A_{q q, Q}^{\mathrm{PS}}$, where for the $\{Q q\}$ - term the operator couples to the heavy quark and for the $\{q q, Q\}$-term to a light quark.

\section{Renormalization}

We work in Feynman-gauge and use dimensional regularization in $D=4+\varepsilon$ dimensions, applying the $\overline{\mathrm{MS}}$-scheme, if not stated otherwise. Renormalization proceeds in four steps, which we will

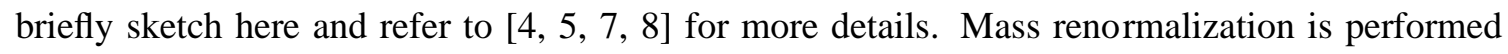
in the on-shell scheme [11], whereas for charge renormalization we use the $\overline{\mathrm{MS}}$-scheme. The remaining singularities are of the ultraviolet and collinear type. The former are renormalized via the operator $Z$-factors, whereas the latter are removed via mass factorization through the transition functions $\Gamma$. After coupling- and mass renormalization, the renormalized heavy flavor OMEs are then obtained via

$$
A=Z^{-1} \hat{A} \Gamma^{-1},
$$

where quantities with a hat are unrenormalized. Note, that in the singlet case operator mixing occurs and hence Eq. (3.1) should be read as a matrix equation, contrary to the NS-case. The Zand $\Gamma$-factors can be expressed in terms of the anomalous dimensions of the twist -2 operators in all orders in the strong coupling constant $a_{s}$, cf. [面] up to $O\left(a_{s}^{3}\right)$. For this purpose, we adopt the convention

$$
\gamma=\mu \partial \ln Z(\mu) / \partial \mu
$$

From Eqs. (3.1 3.2) one can then infer that for operator renormalization and mass factorization at $O\left(a_{s}^{3}\right)$, the anomalous dimensions up to NNLO, [9], together with the 1-loop heavy flavor OMEs up to $O\left(\varepsilon^{2}\right)$ and the 2-loop heavy OMEs up to $O(\varepsilon)$ are needed. Higher orders in $\varepsilon$ enter since they multiply $Z$ - and $\Gamma$-factors containing poles in $\varepsilon$. This has been worked out in some detail in Ref. 叫, where we presented the $O(\varepsilon)$ terms $\bar{a}_{Q g}^{(2)}, \bar{a}_{q q, Q}^{(2), N S}$ and $\bar{a}_{Q q}^{(2) \mathrm{PS}}$ in the unpolarized case. The terms $\bar{a}_{g g, Q}^{(2)}$ and $\bar{a}_{g q, Q}^{(2)}$ were given in Refs. [8]. Thus all terms needed for the renormalization at 3-loops in the unpolarized case are known by now. 


\section{Moments of the Massive OMEs at three Loops}

The heavy flavor OMEs at $O\left(a_{s}^{3}\right)$ are given by 3-loop self-energy type diagrams, which contain a local operator insertion. The external massless particles are on-shell. The heavy quark mass sets the scale and the spin of the local operator is given by the Mellin-variable $N$. The steps for the calculation are the following: We use QGRAF, [12], for the generation of diagrams. Approximately 100 diagrams contribute for each case at hand. For the calculation of the color factors we use [13]. After undoing the contraction of the operators with the light-like vector $\Delta,\left(\Delta^{2}=0\right)$, the diagrams are genuinely given as tensor integrals. Applying a projector then provides the results for the diagrams for the specific (even) Mellin moment under consideration. The diagrams are further translated into a form, which is suitable for the program MATAD [14], through which the expansion in $\varepsilon$ is performed and the corresponding massive three-loop tadpole-type diagrams are calculated. We have implemented all these steps into a FORM-program, cf. [15], and use TFORM, [16], for parts of the calculation. We checked our procedures against various complete two-loop results and certain scalar 3-loop integrals and found full agreement.

\section{Results}

Applying Eq. (3.1), one can predict the pole structure of the unrenormalized results and thus the logarithmic terms of the renormalized OMEs. Since we consider only terms involving at least one heavy quark, we adopt the following notation for the anomalous dimensions

$$
\hat{\gamma} \equiv \gamma\left(n_{f}+1\right)-\gamma\left(n_{f}\right) \text {. }
$$

As an example, we show the structure of the renormalized result in the PS case, where all renormalization constants are taken at $n_{f}$ flavors.

$$
\begin{aligned}
A_{Q(q) q}^{(3), \mathrm{PS}}= & \frac{\hat{\gamma}_{q g}^{(0)} \gamma_{g q}^{(0)}}{48}\left\{\gamma_{g g}^{(0)}-\gamma_{q q}^{(0)}+4\left(n_{f}+1\right) \beta_{0, Q}+6 \beta_{0}\right\} \ln ^{3}\left(\frac{m^{2}}{\mu^{2}}\right)+\left\{\frac{\hat{\gamma}_{q q}^{(1), \mathrm{PS}}}{2}\left(\left(n_{f}+1\right) \beta_{0, Q}-\beta_{0}\right)\right. \\
& \left.+\frac{\hat{\gamma}_{q g}^{(0)}}{8}\left(\left(n_{f}+1\right) \hat{\gamma}_{g q}^{(1)}-\gamma_{g q}^{(1)}\right)-\frac{\gamma_{g q}^{(0)} \hat{\gamma}_{q g}^{(1)}}{8}\right\} \ln ^{2}\left(\frac{m^{2}}{\mu^{2}}\right)+\left\{\frac{\hat{\gamma}_{q q}^{(2), \mathrm{PS}}}{2}-2 a_{Q q}^{(2), \mathrm{PS}} \beta_{0}-\frac{\gamma_{g q}^{(0)}}{2} a_{Q g}^{(2)}\right. \\
& \left.-\zeta_{2} \frac{\hat{\gamma}_{q g}^{(0)} \gamma_{g q}^{(0)}}{16}\left(\gamma_{g g}^{(0)}-\gamma_{q q}^{(0)}+4\left(n_{f}+1\right) \beta_{0, Q}+6 \beta_{0}\right)+\frac{n_{f}+1}{2} \hat{\gamma}_{q g}^{(0)} a_{g q, Q}^{(2)}\right\} \ln \left(\frac{m^{2}}{\mu^{2}}\right)+\gamma_{g q}^{(0)} \bar{a}_{Q g}^{(2)} \\
& +\zeta_{3} \frac{\gamma_{g q}^{(0)} \hat{\gamma}_{q g}^{(0)}}{48}\left(\gamma_{g g}^{(0)}-\gamma_{q q}^{(0)}+4 n_{f} \beta_{0, Q}+6 \beta_{0}\right)+\frac{\zeta_{2}}{16}\left(-4 n_{f} \beta_{0, Q} \hat{\gamma}_{q q}^{(1), \mathrm{PS}}+\hat{\gamma}_{q g}^{(0)} \gamma_{g q}^{(1)}\right) \\
& +4\left(\beta_{0}+\beta_{0, Q}\right) \bar{a}_{Q q}^{(2), \mathrm{PS}}+C_{F}\left(-\left(4+\frac{3}{4} \zeta_{2}\right) \hat{\gamma}_{q g}^{(0)} \gamma_{g q}^{(0)}-4 \hat{\gamma}_{q q}^{(1), \mathrm{PS}}+12 a_{Q q}^{(2), \mathrm{PS}}\right) \\
& -\left(n_{f}+1\right) \hat{\gamma}_{g g}^{(0)} \bar{a}_{g q, Q}^{(2)}+a_{Q(q) q}^{(3), \mathrm{PS}} .
\end{aligned}
$$

Here, the terms $a_{i j}^{(2)}$ denote the constant terms in $\varepsilon$ of the 2-loop OMEs $\hat{A}_{i j}$ and $\beta_{i}$ are the expansion coefficients of the $\beta$-function. The subscript $Q$ refers to contributions due to heavy quarks only, cf. [由, 5, 7], and $\zeta_{i}$ is the Riemann $\zeta$-function at values $i$. All quantities in Eq. (5.2) are known for general values of $N$, except for $a_{Q(q) q}^{(3)}$, which is the genuine 3-loop contribution and remains to be 
calculated. Note, that it is not possible to factor out $n_{f}+1$, not even in the triple pole term. This is due to the interplay of the prescription for coupling constant renormalization we have adopted, cf. [ [ [7], and the fact that the transition functions $\Gamma$ apply to sub-graphs containing massless lines only.

We have calculated the OMEs $A_{Q(q) q}^{\mathrm{PS},(3)}, A_{q q, Q}^{\mathrm{NS},+,(3)}$ and $A_{g q, Q}^{(3)}$ for $N=2 \ldots 12$, using MATAD. All pole terms agree with the general structure derived from renormalization. As an example, we show the constant term after renormalization of the PS-OME for $N=2$

$$
\begin{aligned}
& \left.A_{Q(q) q}^{(3), \mathrm{PS}}\right|_{\mu^{2}=m^{2}} ^{N=2}=\left(\frac{2048}{81} \zeta_{3}-\frac{86480}{2187}\right) C_{F} T_{F}^{2} n_{f}+\left(-\frac{3584}{81} \zeta_{3}+\frac{53144}{2187}\right) C_{F} T_{F}^{2}+\left(\frac{64}{9} B_{4}-\frac{128}{5} \zeta_{2}^{2}\right. \\
& \left.+\frac{1280}{27} \zeta_{3}+\frac{830}{2187}\right) C_{F} C_{A} T_{F}+\left(-\frac{128}{9} B_{4}+\frac{128}{5} \zeta_{2}^{2}-\frac{9536}{81} \zeta_{3}+\frac{95638}{729}\right) C_{F}^{2} T_{F} .
\end{aligned}
$$

The moments we obtain for $N=10,12$ for the corresponding 3-loop anomalous dimensions are shown in Table 1. These results, as well as the moments we obtain for lower values of $N$, agree with the results of Refs. [9]. A check for the constant terms is provided by the sum rule in Eq. (2.2), which is obeyed. Additionally, we find for all moments that the terms proportional to $\zeta_{2}$ disappear after renormalization, which is a general observation made in many $D \rightarrow 4$ calculations. The term $B_{4}$ in Eq. (5.3) is given by

$$
\mathrm{B}_{4}=-4 \zeta_{2} \ln ^{2} 2+\frac{2}{3} \ln ^{4} 2-\frac{13}{2} \zeta_{4}+16 \mathrm{Li}_{4}\left(\frac{1}{2}\right)
$$

and appears in all OMEs we calculated. Since it does not appear in the light-flavor Wilson coeffi-

\begin{tabular}{|c|c|}
\hline $\mathrm{N}$ & $\hat{\gamma}_{g q}^{(2)} / T_{F} / C_{F}$ \\
\hline 10 & $\frac{1218139408}{363862125} T_{F}\left(1+2 n_{f}\right)+\frac{7168}{495}\left(C_{A}-C_{F}\right) \zeta_{3}-\frac{18846629176433}{11767301122500} C_{A}+\frac{529979902254031}{323600780868750} C_{F}$ \\
\hline 12 & $\frac{13454024393417}{5222779912350} T_{F}\left(1+2 n_{f}\right)+\frac{5056}{429}\left(C_{A}-C_{F}\right) \zeta_{3}-\frac{64190493078139789}{48885219979596000} C_{A}+\frac{1401404001326440151}{3495293228541114000} C_{F}$ \\
\hline $\mathrm{N}$ & $\hat{\gamma}_{q q}^{(2), \mathrm{NS},+} / T_{F} / C_{F}$ \\
\hline 10 & $-\frac{27995901056887}{1497656506500} T_{F}\left(1+2 n_{f}\right)+\frac{192880}{693}\left(C_{F}-C_{A}\right) \zeta_{3}-\frac{9007773127403}{97250422500} C_{A}-\frac{75522073210471127}{307518802668000} C_{F}$ \\
\hline 12 & $\begin{array}{l}-\frac{65155853387858071}{3290351344780500} T_{F}\left(1+2 n_{f}\right)+\frac{13549568}{45045}\left(C_{F}-C_{A}\right) \zeta_{3}-\frac{25478252190337435009}{263228107582440000} C_{A} \\
-\frac{35346062280941906036867}{131745667845011220000} C_{F}\end{array}$ \\
\hline $\mathrm{N}$ & $\hat{\gamma}_{q q}^{(2), \mathrm{PS}} / T_{F} / C_{F}$ \\
\hline 10 & $-\frac{265847305072}{420260754375} T_{F}\left(1+2 n_{f}\right)+\frac{50176}{27225}\left(C_{F}-C_{A}\right) \zeta_{3}-\frac{1028766412107043}{1294403123475000} C_{A}+\frac{839864254987192}{485401171303125} C_{F}$ \\
\hline 12 & $\begin{array}{l}-\frac{2566080055386457}{5703275664286200} T_{F}\left(1+2 n_{f}\right)+\frac{49928}{39039}\left(C_{F}-C_{A}\right) \zeta_{3}-\frac{69697489543846494691}{83039693672007072000} C_{A} \\
+\frac{86033255402443256197}{54806197823524667520} C_{F}\end{array}$ \\
\hline
\end{tabular}
cients, cf. [3], it occurs as a genuine mass effect.

Table 1: Results for $N=10,12$ for the 3-loop anomalous dimensions 


\section{Conclusions and Outlook}

We calculated the heavy OMEs $A_{q q, Q}^{(3), \mathrm{NS},+}, A_{Q q}^{(3), \mathrm{PS}}$ and $A_{g q, Q}^{(3)}$ for even Mellin-moments $N=2 \ldots 12$ using MATAD and showed first results. This confirms for the first time in an independent calculation the moments of the fermionic parts of the corresponding 3-loop anomalous dimensions, [9]. We expect results for the remaining terms $A_{g g, Q}^{(3)}$ and $A_{Q g}^{(3)}$ in the near future, thus enabling us to calculate fixed moments of the heavy flavor Wilson coefficients in the asymptotic limit $Q^{2} \gg m^{2}$.

Acknowledgments. The work was supported in part by SFB-TR-9: Computergestützte Theoretische Teilchenphysik and EU-network HEPTOOLS. We would like to thank M. Steinhauser and J. Vermaseren for useful discussions and M. Steinhauser for a FORM 3.0 compatible form of the code MATAD.

\section{References}

[1] E. Laenen et al., Nucl. Phys. B 392 (1993) 162; S. Riemersma, J. Smith and W. L. van Neerven, Phys. Lett. B 347 (1995) 143,[hep-ph/9411431].

[2] S. I. Alekhin and J. Blümlein, Phys. Lett. B 594 (2004) 299 [hep-ph/0404034].

[3] J. A. M. Vermaseren, A. Vogt and S. Moch, Nucl. Phys. B 724 (2005) 3, [hep-ph/0504242].

[4] M. Buza, et al., Nucl. Phys. B 472 (1996) 611 [hep-ph/9601302].

[5] I. Bierenbaum, J. Blümlein and S. Klein, Nucl. Phys. B 780 (2007) 40. [hep-ph/0703285]; Phys. Lett. B 648 (2007) 195 [hep-ph/0702265].

[6] J. Blümlein, et al. Nucl. Phys. B 755 (2006) 272 [hep-ph/0608024].

[7] I. Bierenbaum, et al., Nucl. Phys. B 803 (2008) 1 [hep-ph/0803.0273]; PoS ACAT2007 (2007) 082 [math-ph/0707.4659].

[8] I. Bierenbaum, J. Blümlein and S. Klein, Acta Phys. Polon. B 39 (2008) 1531 [hep-ph/0806.0451]; Nucl. Phys. Proc. Suppl. 183 (2008) 162 and in preparation, [hep-ph/0806.4613].

[9] S. A. Larin, T. van Ritbergen and J. A. M. Vermaseren, Nucl. Phys. B 427 (1994) 41; S. A. Larin, et al., Nucl. Phys. B 492 (1997) 338 [hep-ph/9605317]; A. Retey and J. A. M. Vermaseren, Nucl. Phys. B 604 (2001) 281 [arXiv:hep-ph/0007294]; J. Blümlein and J. A. M. Vermaseren, Phys. Lett. B 606 (2005) 130 [arXiv:hep-ph/0411111]; S. Moch, J. A. M. Vermaseren and A. Vogt, Nucl. Phys. B 688 (2004) 101; [hep-ph/0403192]; Nucl. Phys. B 691 (2004) 129 [hep-ph/0404111].

[10] M. Buza, et al., Eur. Phys. J. C 1 (1998) 301 [hep-ph/9612398].

[11] D. J. Broadhurst, et al. Z. Phys. C 48 (1990) 673; 52 (1991) 111.

[12] P. Nogueira, J. Comput. Phys. 105 (1993) 279.

[13] T. van Ritbergen, A. N. Schellekens and J. A. M. Vermaseren, Int. J. Mod. Phys. A 14 (1999) 41 [hep-ph/9802376].

[14] M. Steinhauser, Comput. Phys. Commun. 134 (2001) 335 [hep-ph/0009029] and MATAD 3.0.

[15] J. A. M. Vermaseren, New features of FORM, arXiv:math-ph/0010025.

[16] M. Tentyukov and J. A. M. Vermaseren, arXiv:hep-ph/0702279. 